\title{
Effect of Probiotic and Green Tea Mouth Rinse Against Salivary Streptococcus mutans: A Randomized Controlled Trial
}

\author{
Manisha Koul*, Arpan Debnath, Alok Pandey and Priyanka D
}

Department of Public Health Dentistry, India

ISSN: 2637-7764

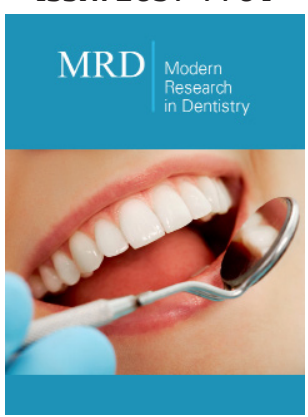

*Corresponding author: Manisha Koul, Department of Public Health Dentistry, Krishnadevaraya College of Dental Sciences, Hunasamarnahalli, International Airport Road, Bangalore, India

Submission: 侮 June 02, 2019

Published: 监July 05, 2019

Volume 4 - Issue 2

How to cite this article: Manisha $\mathrm{K}$ Arpan D, Alok P, Priyanka D. Effect of Probiotic and Green Tea Mouth Rinse Against Salivary Streptococcus mutans: A Randomized Controlled Trial. Mod Res Dent. 4(2). MRD.000584.2019.

DOI: 10.31031/MRD.2019.04.000584

Copyright@ Manisha Koul, This article is distributed under the terms of the Creative Commons Attribution 4.0 International License, which permits unrestricted use and redistribution provided that the original author and source are credited.

\begin{abstract}
Introduction: Probiotics have been used to provide benefit to general health, but their effect on oral health especially on dental caries is yet to be explored in detail. Green tea is exclusively known for antioxidant and antimicrobial properties. Thus, the aim of the present study was to evaluate the anti-microbial efficacy of probiotic and green tea mouth rinse on salivary Streptococcus mutans count.
\end{abstract}

Methods: A triple-blinded, concurrent parallel group randomized trial was conducted which included 45 subjects, aged 21-25years. The subjects were randomly divided into three groups namely probiotic group, green tea group and chlorhexidine group. The participants were instructed to rinse their mouth once daily in the morning with the allocated mouth rinse. Unstimulated Saliva samples were collected at baseline, 30minutes post rinse and after 15 days of intervention. Microbial analysis was done to record Streptococcus mutans count.

Results: There was no statistically significant difference in reduction of microbial count of Streptococcus mutans between green tea and chlorhexidine mouth rinse after 15 days specifying both are equally effective in reducing $S$. Mutans count. However, probiotic group showed least antibacterial effect.

Conclusion: Green tea proved to be more effective in reducing Streptococcus mutans than probiotic mouth rinse. Also, the antibacterial effect of green tea was similar to that of chlorhexidine mouth rinse. Thus, green tea mouth rinse was found to be a successful alternate to chlorhexidine mouth rinse.

Keywords: Probiotic; Green tea; Streptococcus mutans; Mitis salivarius bacitracin agar; Mouth rinse

\section{Introduction}

Dental caries is an ongoing disease with multifactorial etiology. Microorganisms like Streptococcus mutans and Lactobacillus have been shown to play a pivotal role in dental caries. Thus, reducing microbial count of these cariogenic microorganisms causes a significant decrease in dental caries. The term probiotic, as an antonym to the term antibiotic, was originally proposed in 1965 by Lilley and Stillwell [1]. Probiotics are food ingredients with a sufficient number of viable microorganisms that are beneficial to the health of the host. They are especially known for improving intestinal microbial health and have been used extensively in yoghurt, cheese, capsules [2]. As the mouth represents the first part of the gastrointestinal tract there is every reason to believe that at least some probiotic mechanisms may also play a role in that part of the system [3]. Several investigations have shown reduction in the number of Mutans Streptococci in saliva after consumption of various probiotic products [46]. Tea is one of the most commonly consumed beverages in the world. Among various types of tea available, Green tea is known for its antimicrobial, anti-inflammatory and antioxidant properties. Certain studies have reported anticariogenic effect of green tea [7,8]. Using mouthwash is an effective and safe method for maintaining oral hygiene. Ample research 
has been done on antibacterial effect of green tea mouth rinse. However, data supporting the action of probiotic in the form of mouth rinse against Streptococcus mutans is still sparse. Hence, the present study was conducted to evaluate the anti-microbial efficacy of probiotic and green tea mouth rinse on salivary Streptococcus mutans count and compare it against chlorhexidine mouth rinse (control).

\section{Material and Methods}

A triple-blinded concurrent parallel arm randomized controlled trail was conducted in the Department of public health dentistry, Krishnadevaraya College of Dental Sciences and Hospital, Bengaluru. Simple random sampling technique was used to select 45 subjects. Sample size calculation was based on the proportion of reduction in colony counts as observed in pilot study.

The required sample size was estimated using the following formula:

$$
N=\frac{\left(Z_{\alpha}\right)^{2} p q}{L^{2}}
$$

Where,

$\mathrm{Z}_{\alpha}=\mathrm{Z}$ value for alpha error at $95 \% \mathrm{CI}=1.96$.

$\mathrm{P}=$ The proportion of reduction in colony counts as observed in pilot study (in \%)=90.

$$
\mathrm{q}=1 \mathrm{p}(\text { in } \%)=10 \text {. }
$$

$\mathrm{L}=$ Permissible error in estimation of $\mathrm{p}=10 \%$ of $\mathrm{p}=10 \%$ of $90=9$.

Hence sample size of 45 subjects with 15 subjects in each group were selected among students of the dental college. Subjects aged between 20-25 years, who fulfilled the following eligibility criteria, were selected.

\section{A. Inclusion criteria}

a. Female subjects aged between 20-25 years.

b. Adults with DMFT $>3$.

c. Adults with no recent antibiotic therapy (within 2 weeks).

\section{B. Exclusion criteria}

a. Known history of allergy to any mouth rinse or drug.

b. Any systemic illness.

c. Using any other commercially available mouth rinse.

d. History of fluoride treatment in the past 2 weeks.

The study population consisted of undergraduate dental students of Krishnadevaraya College of Dental Sciences, Bangalore. To avoid the confounding effect of diet, the subjects residing in hostel having almost same dietary pattern were chosen. The study participants were also instructed to use non-fluoridated toothpaste to ensure anticariogenic effect of fluoride was eliminated. The ethical clearance was obtained from the Institutional Review Board of Krishnadevaraya College of Dental Sciences.

\section{Randomization}

Using random allocation rule, 45 participants were randomly divided into three groups

a. Group A: probiotic mouth rinse

b. Group B: green tea mouth rinse

c. Group C: chlorhexidine $0.2 \%$ mouth rinse

Cards with group names were kept in sealed envelopes, and all the participants were asked to pick up the sealed envelopes. The group allocation was disclosed by principal investigator after opening the envelopes.

\section{Intervention}

Preparation of probiotic mouth rinse: Probiotic mouth rinse was prepared using commercially available probiotic sachet (Darolac $\AA$ ). 1gm probiotic powder contains 1.25 billion freeze dried bacterial combination comprised of Lactobacillus acidophilus, Lactobacillus rhamnosus, Bifidobacterium longum, and Saccharomyces boulardii. The sachet contents were dissolved in $10 \mathrm{ml}$ of distilled water which was used as a mouth rinse.

Preparation of green tea mouth rinse: Fresh green tea, available in the form of green tea dip bags with packing date less than one month was procured from local market. Green tea dip bag containing 2 grams of green tea was dipped in $100 \mathrm{ml}$ of warm distilled water for five minutes.

Preparation of chlorhexidine mouth rinse: $0.2 \%$ commercially available chlorhexidine was diluted with $10 \mathrm{ml}$ of distilled water (1:1 ratio) (Figure 1).

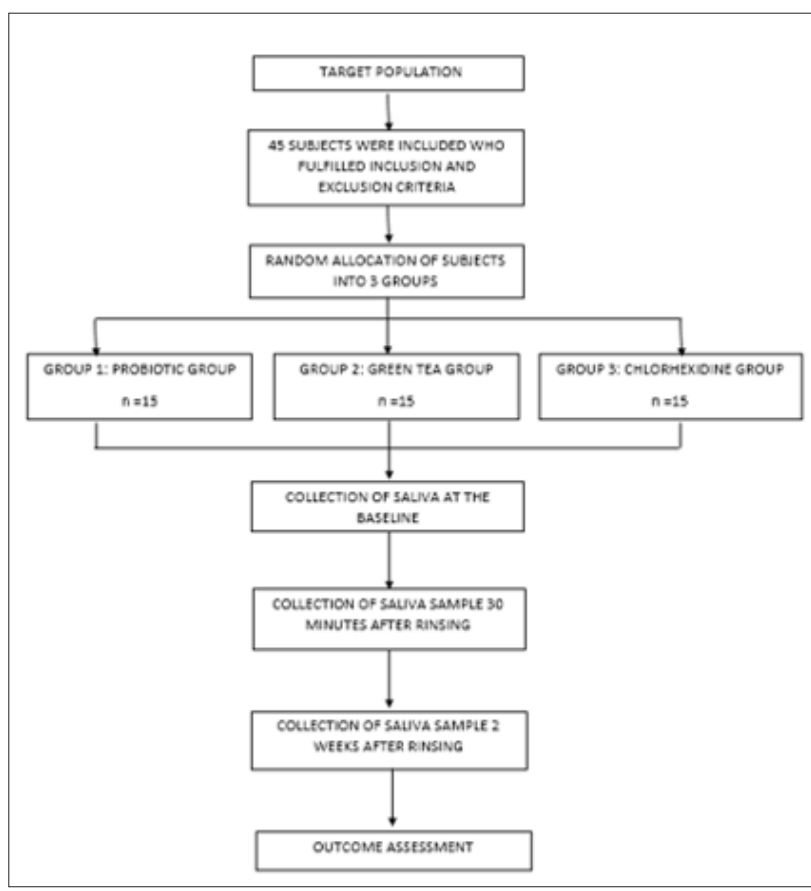

Figure 1: Flow diagram of study protocol according to consort. 
The freshly prepared mouth rinses were dispensed in plastic screw capped containers for the participants $(10 \mathrm{ml}$ for each participant). Each subject was instructed to swish with $10 \mathrm{ml}$ mouth rinse for 1 minute before spitting it. Study participants were refrained from eating or drinking two hours before using mouth rinse. The primary examiner (author 1) selected the students, obtained informed consent from them, did primary screening for inclusion and exclusion criteria, followed by oral prophylaxis of the study participants. The oral prophylaxis was done in all subjects to make all groups comparable at the baseline, before the intervention. Following oral prophylaxis, the study participants were recalled to the department next day morning. The second examiner (author 2) administered the mouth rinse without the knowledge of the type of mouth rinse being administered, collected unstimulated saliva samples before rinsing, 30 minutes after rinsing on the $1^{\text {st }}$ day and after 2 weeks. The microbial analysis was done and mean values of $S$. Mutans counts were noted down. The microbiologist was also blinded, thus ensuring triple blinded design of the study.

\section{Microbial analysis}

After collecting saliva samples, $100 \mu$ of each saliva sample was transferred to $10 \mathrm{ml}$ of sterile Brain Heart Infusion Agar (BHA) broth followed by spreading of saliva samples on a sterile Mitis Salivarius Bacitracin Agar media plate. Inoculated culture media were incubated for 48 hours at $37^{\circ} \mathrm{C}$ in a candle extinction jar. $S$. Mutans colonies on the agar plate were identified as small convex deep blue colonies. The colonies on plates were counted using Digital colony counter and the colony-forming units/ml (CFU/ml) of saliva were calculated.

\section{Statistical analysis}

The statistical analysis was performed using SPSS Version 22.0, to compare the groups at baseline, after 30 minutes of using mouth rinses and after 2 weeks of using the mouth rinses. Shapiro Wilk test was done to assess the normality of the data. Results were expressed as mean \pm standard deviation (SD). ANOVA was applied to compare intra and inter group mean differences. Post hoc analysis using Tukey's multiple comparison test was applied to assess the significance of changes among the groups. A ' $p$ ' value of $<0.05$ was considered as significant.

\section{Result}

The mean age of the study participants in Group A, Group B and Group C was $22.47 \pm 1.25,21.53 \pm 1.11$ and $22.66 \pm 1.31$ respectively. A statistically significant reduction in mean Streptococcus mutans count was found in all three experimental groups, 30 min post rinse and after 2 weeks of intervention (Table 1). Also, at the baseline, all the three groups were similar with no statistically significant difference among them indicating baseline comparability among the three groups. A statistically significant reduction of $S$. Mutans count was seen in all the three groups 30 minutes post rinsing as well as 2 weeks after intervention. (Table 2) (Figure 2). Table 3 shows that when pairwise comparisons were done, a statistically significant reduction in $S$. Mutans count was more profound in chlorhexidine group as compared to probiotic group, 2 weeks after intervention. Also, in comparison to probiotic group, greater reduction in S. Mutans count was seen in green tea group, which was statistically significant. There was no statistically significant difference between green tea and chlorhexidine group indicating that both are equally effective against $S$. Mutans. However, probiotic mouth rinse showed least antibacterial effect (Figures 3-5).

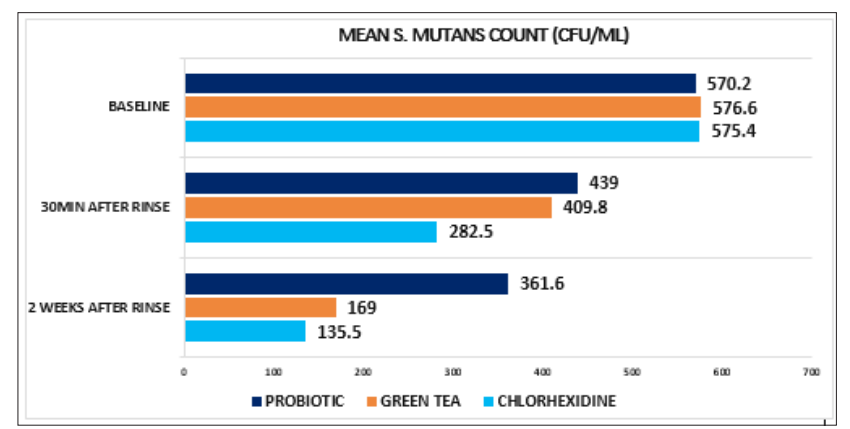

Figure 2: Intergroup Comparison of the three Mouthrinses.

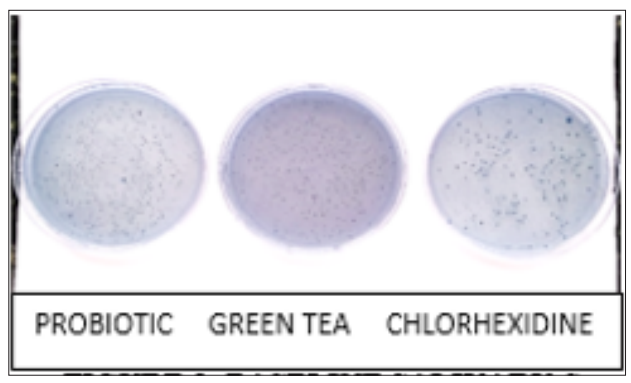

Figure 3: Baseline salivary S. mutans colonies on MSB agar plate.

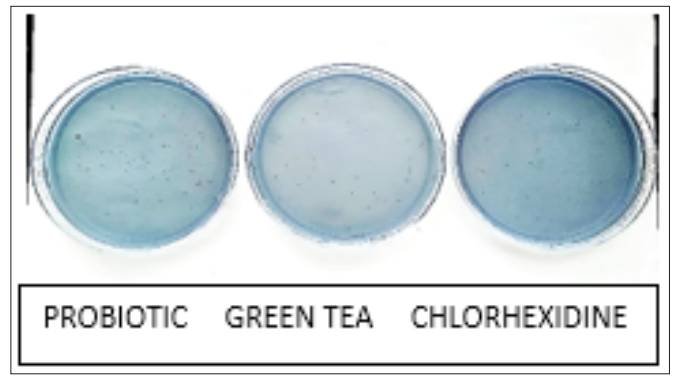

Figure 4: 30 minutes post rinse salivary S. mutans colonies on MSB agar plate.

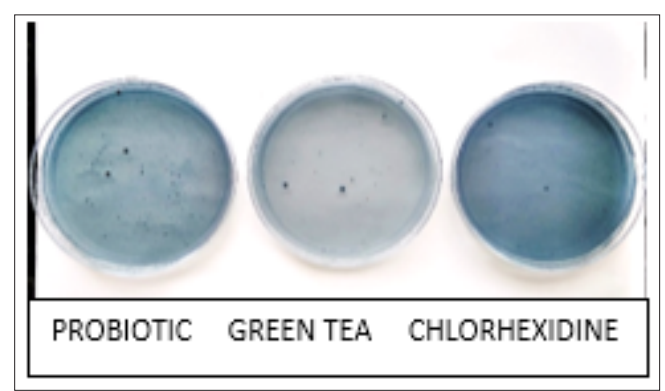

Figure 5: 2 weeks post rinse salivary S. mutans colonies on MSB agar plate. 
Table 1: Intragroup comparison of the three mouthrinses.

\begin{tabular}{|c|c|c|c|c|c|}
\hline Group & & $\begin{array}{c}\text { Mean S. mutans Count (CFU/ml } \\
\times 103) \text { Mean } \pm \text { SD }\end{array}$ & $95 \% \mathrm{CI}$ & F Value & P Value \\
\hline \multirow{3}{*}{ Probiotic } & Baseline & $570.2 \pm 62.15$ & $494.6-593.6$ & \multirow{3}{*}{4.627} & \multirow{3}{*}{$0.0028^{*}$} \\
\hline & 30min Post Rinse & $439 \pm 45.31$ & $406.6-471.4$ & & \\
\hline & After 2 Weeks & $361.6 \pm 35.03$ & $336.5-386.7$ & & \\
\hline \multirow{3}{*}{ Green tea } & Baseline & $576.6 \pm 71.4$ & $525.5-627.7$ & \multirow{3}{*}{8.269} & \multirow{3}{*}{$<0.0001^{*}$} \\
\hline & 30min Post Rinse & $409.8 \pm 70.12$ & $359.6-460$ & & \\
\hline & After 2 Weeks & $169 \pm 30.11$ & $157.5-182.5$ & & \\
\hline \multirow{3}{*}{ Chlorhexidine } & Baseline & $575.4 \pm 75.44$ & 523.4-631.4 & \multirow{3}{*}{10.19} & \multirow{3}{*}{$<0.0001^{*}$} \\
\hline & 30min Post Rinse & $282.5 \pm 74.53$ & $229.2-335.8$ & & \\
\hline & After 2 Weeks & $135.5 \pm 33.32$ & $111.7-159.3$ & & \\
\hline
\end{tabular}

One-way ANOVA

Table 2: Intergroup comparison of the three Mouthrinses at the baseline, 30 minutes post rinse and 2 weeks after intervention.

\begin{tabular}{|c|c|c|c|c|c|}
\hline & Groups & $\begin{array}{c}\text { Mean S. mutans Count (CFU } / \mathrm{ml} \times 103) \\
\text { Mean } \pm \text { SD }\end{array}$ & $95 \%$ CI & F Value & $P$ Value \\
\hline \multirow{3}{*}{ Baseline } & Probiotic & $570.2 \pm 62.15$ & 494.6-593.6 & \multirow{3}{*}{11.05} & \multirow{3}{*}{0.3121} \\
\hline & Green tea & $576.6 \pm 71.4$ & $525.5-627.7$ & & \\
\hline & Chlorhexidine & $575.4 \pm 75.44$ & 523.4-631.4 & & \\
\hline \multirow{3}{*}{$30 \mathrm{~min}$ post rinse } & Probiotic & $439 \pm 45.31$ & $406.6-471.4$ & \multirow{3}{*}{15.34} & \multirow{3}{*}{$0.0289 *$} \\
\hline & Green tea & $409.8 \pm 70.12$ & $359.6-460$ & & \\
\hline & Chlorhexidine & $282.5 \pm 74.53$ & $229.2-335.8$ & & \\
\hline \multirow{3}{*}{ After 2 weeks } & Probiotic & $361.6 \pm 35.03$ & $336.5-386.7$ & \multirow{3}{*}{18.63} & \multirow{3}{*}{$<0.0001^{*}$} \\
\hline & Green tea & $169 \pm 30.11$ & $147.5-190.5$ & & \\
\hline & Chlorhexidine & $135.5 \pm 33.32$ & $111.7-159.3$ & & \\
\hline
\end{tabular}

One way ANOVA.

\section{Discussion}

The present study demonstrated a significant reduction of Streptococcus mutans following daily mouth rinse with green tea and probiotic mouth rinse. There was no loss due to follow up and no adverse effects were reported by the participants. Mouth rinses are widely used to improve oral hygiene and thus, have been of great interest to dental researchers. Most of the mouth rinses are available 'over the counter' and the most popular among them is chlorhexidine mouth rinse which is considered to be the gold standard.

A large number of microorganisms are inhibited by Chlorhexidine gluconate mouthwash as it exhibits both anti-plaque and antibacterial properties. Its superior anti-plaque activity is attributed to its substantivity and pin-cushion effect. However, the long-term use of chlorhexidine produces brown staining, oral mucosal ulceration, unilateral/bilateral parotid swelling $[9,10]$. Also, many mouth rinses contain alcohol as one of their ingredients which may produce burning sensation. Thus, the side-effects associated with various commercially available mouth rinses have encouraged the search for alternative agents [11]. The good old concept of bacteriotherapy which relies upon restoration of the microbial ecological balance, rather than elimination of the disease associated species may be explored in preventive dentistry [12]. These include the use of probiotics to promote growth of health associated bacteria to compete against pathogenic bacteria. Probiotic bacteria (Lactobacilli and Bifidobacteria) protect the oral cavity by competing with the oral pathogens for nutrients, growth factors and site of adhesion. Once adhered to the oral cavity, probiotic bacteria aggregate followed by inhibiting the adhesion of the harmful microorganisms by producing bacteriocins or other antimicrobial compounds such as acids or peroxides [13]. 
Green tea has been considered a healthy beverage since ancient times [14]. Tea specially, the green tea contains a significant number of polyphenols, catechins, tannin. Previous studies have reported polyphenols and catechins like epicatechin, gallocatechin (GC), epigallocatechin (EGC) and epigallocatechin gallate (EGCG) exert an antimicrobial effect $[14,15]$. It has been proposed that microbial cytoplasmic membrane damage results from the generation of hydrogen peroxide by EGCG [15]. In the present study, it was found that all the three mouth rinses demonstrated reduction in Streptococcus mutans count but chlorhexidine group showed a greater reduction of $S$. Mutans count followed by green tea and probiotic mouth rinse (Table $1 \& 2$ ). Also, there was no statistically significant difference in reduction of microbial count of $S$. mutans between green tea and chlorhexidine mouth rinse group at the end of 15 days indicating both are equally effective against Streptococcus mutans (Table 3).

Table 3: Intergroup comparison of mouth rinses 30min post rinse and 2 weeks post mouth rinse.

\begin{tabular}{|c|c|c|}
\hline & $\begin{array}{l}\text { Mean difference in reduction of Mutans Streptococci Count } \\
\qquad(\mathrm{CFU} / \mathrm{ml} \times 103)\end{array}$ & P Value \\
\hline \multicolumn{3}{|c|}{$30 \mathrm{~min}$ post rinse } \\
\hline Probiotic vs Chlorhexidine & -156.5 & $<0.001^{* * *}$ \\
\hline Green Tea vs Chlorhexidine & -127.3 & $<0.001^{* * *}$ \\
\hline Probiotic vs Green Tea & -63.9 & 0.091 \\
\hline \multicolumn{3}{|c|}{ After 2 weeks } \\
\hline Probiotic vs Chlorhexidine & -226.1 & $<0.001^{* * *}$ \\
\hline Green Tea vs Chlorhexidine & -33.5 & 0.732 \\
\hline Probiotic vs Green Tea & -192.6 & $<0.001^{* * *}$ \\
\hline
\end{tabular}

Tukey's multiple comparison test.

However, probiotics were found to have least antibacterial effect. Precise valid comparisons of antibacterial effect of probiotics with other studies could not be done due to variation in form of probiotic administered, strain of probiotic used or the time period of intervention. The studies done by Yadav et al. [16] and Tehrani el al. [17] found a statistically significant reduction of Streptococcus mutans level decreased significantly in probiotic group after intervention $[16,17]$.

In contrast, a study conducted by Montalto et al. [18] found that the oral administration of probiotics, both in capsules and in liquid form, significantly increases salivary counts of lactobacilli and S. mutans populations were not significantly affected [18]. Green tea showed potent antimicrobial effect similar to chlorhexidine mouth rinse. This finding is similar to study conducted by Awadalla [14] and Singh [19] in which there was a statistically significant difference between salivary Streptococcus mutans count before and after intake of green tea mouth rinse [15,19]. Green tea has certain advantages over probiotics and commercially available chlorhexidine, like it is easily available, cost effective, causes no taste alteration, no staining of teeth, no allergy, no reported side effects. Green tea mouth rinse thus appear to have potential antibacterial effect against Streptococcus mutans and its regular use may prove beneficial in preventing dental caries.

\section{Limitations}

The present study was conducted on a small sample. The study was conducted for a period of 2 weeks. Although the confounding effect of diet and oral hygiene practices were taken care of at the beginning of the study, further extensive longitudinal study on a larger sample size is recommended to elucidate the long-term antibacterial effect of probiotic and green tea mouth rinse against S. mutans more strongly.

\section{Conclusion}

The present study shows that green tea mouth rinse was equally effective as conventional chlorhexidine mouthwash in reduction of $S$. mutans count over 15 days usage. Probiotic mouth rinse exerted minimal antimicrobial effect as compared to green tea and chlorhexidine group. Green tea is a cost-effective, easily available and safe long-term adjunct to oral self-care of patients as it has prophylactic benefits with minimum side effects. Green tea mouth rinse thus appear to have promising antibacterial effect and can be incorporated safely into daily oral hygiene regimen. Further studies are needed to evaluate a dose-response relationship more precisely.

\section{References}

1. Lilly DM, Stillwell RH (1965) Probiotic growth promoting substances produced by microorganisms. Science 147(3659): 747-748.

2. Young RJ, Huffman S (2003) Probiotic use in children. J Pediatr Health Care 17(6): 277-283.

3. Parvez S, Malik KA, Ah Kang S, Kim HY (2006) Probiotics and their fermented food products are beneficial for health. J Appl Microbiol 100(6): 11711185.

4. Näse L, Hatakka K, Savilahti E, Saxelin M, Pönkä A, et al. (2001) Effect of Long-Term Consumption of a Probiotic Bacterium, Lactobacillus rhamnosus GG, in Milk on Dental Caries and Caries Risk in Children. Caries Res 35(6): 412-420.

5. Nikawa H, Makhihira S, Fukushima H, Nishimura H, Ozaki Y, et al. (2004) Lactobacillus reuteri in bovine milk fermented decreases the oral carriage of mutans streptococci. Int J Food Microbiol 95(2): 219-223. 
6. Caglar E, Cildir SK, Ergeneli S, Sandalli N, Twetman S (2006) Salivary mutans streptococci and lactobacilli levels after ingestion of the probiotic bacterium Lactobacillus reuteri ATCC 55730 by straws or tablets. Acta Odontol Scand 64(5): 314-318.

7. Sakanaka S, Kim M, Taniguchi M, Yamamato T (1989) Antibacterial substances in Japanese green tea extract against Streptococcus mutans, a cariogenic bacterium. Agric Biol Chem 53(9): 2307-2311.

8. Cabrera C, Artacho R, Giménez R (2006) Beneficial effects of green tea: A review. J Am Coll Nutr 25(2): 79-99.

9. Flötra L, Gjermo P, Rölla G, Waerhaug J (1971) Side effects of chlorhexidine mouth washes. Scand J Dent Res 79: 119-1125.

10. Mali AM, Behal R, Gilda SS (2012) Comparative evaluation of $0.1 \%$ turmeric mouthwash with $0.2 \%$ chlorhexidine gluconate in prevention of plaque and gingivitis: A clinical and microbiological study. J Ind Soc Periodontol 16(3): 386-391.

11. Vishnu HP (2012) Probiotics and oral health, oral health care-pediatric, research, epidemiology and clinical practices.

12. Purunaik S, Thippeswamy HM, Chavan SS (2014) To evaluate the effect of probiotic mouthrinse on plaque and gingivitis among 15-16-year-old school children of Mysore city, India- randomized controlled trial. Glob J Med Res 14(4): 9-14.

13. Neturi RS, Srinivas R, Simha VB, Sree SY, Chandra shekar T, et al. (2014) Effects of green tea on Streptococcus mutans counts-a randomised control trail. J Clin Diagn Res 8(11): ZC128-30.
14. Awadalla HI, Ragab MH, Bassuoni MW, Fayed MT, Abbas MO (2011) A pilot study of the role of green tea use on oral health. Int J Dent Hyg 9(2): 110-116.

15. Arakawa H, Maeda M, Okubo S, Shimamura T (2004) Role of hydrogen peroxide in bactericidal action of catechin. Biol Pharm Bull 27(3): 277281.

16. Yadav M, Poornima P, Roshan NM, Prachi N, Veena M, et al. (2014) Evaluation of probiotic milk on salivary mutans streptococci count: an in vivo microbiological study. J Clin Ped Dent 39(1): 23-26.

17. Tehrani MH, Akhlaghi N, Talebian L, Emami J, Keyhani SE (2016) Effects of probiotic drop containing Lactobacillus rhamnosus, Bifidobacterium infantis, and Lactobacillus reuteri on salivary Streptococcus mutans and Lactobacillus levels. Contemp Clin Dent 7(4): 469-474.

18. Montalto M, Vastola M, Marigo L, Covino M, Graziosetto R, et al. (2004) Probiotic treatment increases salivary counts of lactobacilli: a doubleblind, randomized, controlled study. Digestion 69(1): 53-56.

19. Singh P, Nagar P, Bharti M, Aminah M (2017) A comparative study between indigenously prepared green tea and green tea in combination with triclosan as mouth rinse in reducing Streptococcus mutans Count in the Children. IJCMR 4(2). 